\title{
Helium Ion Microscopy for the Characterization of Complex 3D Structures in a C/CrC Nanocomposite
}

\author{
Z. Zhou*, M.A.E. Jepson*, X. Liu**, W.M. Rainforth*, and C. Rodenburg* \\ * Department of Engineering Materials, The University of Sheffield, Sheffield, S1 3JD, UK \\ ** Carl Zeiss NTS, Carl-Zeiss-Strasse 56, 73447, Oberkochen, Germany
}

Accurate three-dimensional (3D) nanoscale characterization of materials is the ultimate goal of microscopy. The two major routes towards this using scanning transmission electron microscopy (STEM) are tomography (1 $\mathrm{nm}^{3}$ volume resolution) and depth sectioning. The breakthrough of $\mathrm{C}_{\mathrm{s}}$ correction now allows a routine lateral point resolution of $\sim 0.08 \mathrm{~nm}$ for STEM. However, in the case of complex structures with varying microstructures in depth ranging from a few nanometers to micrometers, the depth resolution is between $4 \mathrm{~nm}$ in the case of imaging single atoms [1], to $\sim 100$ $\mathrm{nm}$ in thicker sample areas. The depth resolution is governed by the wavelength of the source and the convergence angle [1]. The dimensions resolved by STEM are therefore $0.08 \times 0.08 \times(4$ to 100$)$ $\mathrm{nm}^{3}$, giving an analyzed volume of 0.0256 to $0.64 \mathrm{~nm}^{3}$. The former can only be achieved in a small portion of very thin areas, whereas the latter is more representative for the majority of the electron transparent area. The Helium Ion Microscope (HeIM) shows great potential for imaging with both high lateral and depth resolutions due to its small interaction volume near the sample surface [2]. Herein we compare the suitability of HeIM to the STEM for the investigation of complex 3D microstructures.

Images collected in the HeIM are produced using a similar principle to images formed in the scanning electron microscope (SEM), only that the secondary electrons (SEs) are generated by a finely focused He-ion beam (as opposed to an electron beam). The HeIM, however, has a smaller probe size due to a sub-nanometer virtual source size and much shorter wavelength than electrons. Because of this, a lateral resolution of $0.24 \mathrm{~nm}$ has been achieved on a Zeiss Orion ${ }^{\circledR}$ HeIM [3]. Taking the mean escape depth of $1.08 \mathrm{~nm}$ as modeled for carbon by $\mathrm{He}^{+}$irradiation [4], the smallest sampled volume in the HeIM would be $0.0622 \mathrm{~nm}^{3}(0.24 \times 0.24 \times 1.08)$, well within the range that can be achieved when using Cs corrected STEM. Here, this assumption is tested and the information contained in the HeIM image is compared to that obtained in STEM on a nanocomposite consisting of amorphous carbon clusters embedded in a $\mathrm{CrC}$ matrix. The same TEM specimen was imaged using: (1) JEOL 2010 field emission gun (S)TEM with an annular dark field (ADF) detector and a Gatan GIF 2000; (2) Carl Zeiss Orion ${ }^{\circledR}$ Plus HeIM.

Fig. 1a shows the coating microstructure when imaged using STEM/ADF, it reveals multilayers of alternating bright and dark contrast. Fig.1b is the electron energy loss spectrum (EELS) of the region. The absolute thickness of the area can be estimated using EELS by $t / \lambda=\ln \left(\mathrm{I}_{t} / \mathrm{I}_{0}\right), \lambda$ is a total mean free path (MFP) for all transmitted inelastic scattered electrons, $I_{t}$ is the total intensity of the whole spectrum, $I_{0}$ is the intensity of the zero loss peak. $t / \lambda=1.11$ was obtained. Using the inelastic MFP of $200 \mathrm{keV}$ electrons in $\mathrm{C}$ which was estimated to be $116 \mathrm{~nm}$ [5], the specimen thickness is then found to be $127 \mathrm{~nm}$. Closer inspection (Fig. 1c) at a very thin area using high resolution TEM shows amorphous inclusions (3-12 nm size) in a crystalline matrix. The crystalline matrix has been identified as $\mathrm{CrC}$ using $\mathrm{X}$-ray and electron diffraction (not included here). The STEM/ADF image indicates that nanoscale amorphous $\mathrm{C}$ clusters segregate into layers separated by $\mathrm{CrC}(\sim 17 \mathrm{~nm}$ thick), giving a multilayer appearance with a periodicity of $\sim 22 \mathrm{~nm}$. For TEM sample areas thinner 
than $\sim 40 \mathrm{~nm}$, individual $\mathrm{C}$ clusters can be separated. For thicker TEM sample areas, the $\mathrm{C}$ clusters often appear connected to the extent in Fig.1a, where they erroneously appear as continuous layers.

Fig.2a shows the coating microstructure using HeIM. It reveals well separated C clusters in the layer, in contrast to continuous/semi-continuous $\mathrm{C}$ cluster structure revealed by TEM, mainly attributed to overlapping of the clusters through the sample depth. A detailed particle diameter analysis led to an estimation of the mean information depth of $4.8 \mathrm{~nm}$ for the HeIM image. In other words, the HeIM image was formed mainly by SEs escaping from a thin section with a thickness of $4.8 \mathrm{~nm}$.

As a result of the growth process, the $C$ cluster distribution in the $X-Y$ and $Z-Y$ planes are approximately the same where $\mathrm{X}$ is perpendicular to the coating growth direction, $\mathrm{Y}$ is the coating growth direction and $\mathrm{Z}$ is the depth, as shown in Fig.2b. Since the HeIM image signal has been estimated to arise from a depth of $4.8 \mathrm{~nm}$, we can now simulate the STEM data by averaging the intensity of the HeIM image in the X-Y plane over $\mathrm{X}=127 \mathrm{~nm}$, as this may represent a STEM area of $X=4.8 \mathrm{~nm}$ at a thickness of $127 \mathrm{~nm}$. The areas are marked in Fig. 1a and Fig.2a respectively. The slab in Fig.1a and the slice in Fig.2a are illustrated in Fig.2b. Fig.2c shows the matched profiles. This confirms that HeIM has a similar volume resolution to STEM. Therefore, the HeIM can be considered to be a tool for 3D reconstruction of large areas with outstanding depth resolution.

References:

[1] A.Y. Borisevich, A. R. Lupini, S. J. Pennycook, PNAS 103 (9) (2006) 3044

[2] R.Reiche, R.Kaesmaier, R.Rosenkranz, U.Ritter, S.Teichert, S.Leinert. Microscopy and analysis, 23 July (2009) 11.

[3] Zeiss Press Release, Nov-2008. PI Number: 0220-2008-ENG.

[4] R. Ramachandra, B. Griffin, D. Joy, Ultramicroscopy 109 (6) ( 2009) 748.

[5] K Iakoubovskii and K Mitsuishi. J Phys.:Condens. Mater 21(2009) 155402(5pp).

[6] This research was supported by EPSRC UK. C. Rodenburg thanks the Royal Society.

(a)

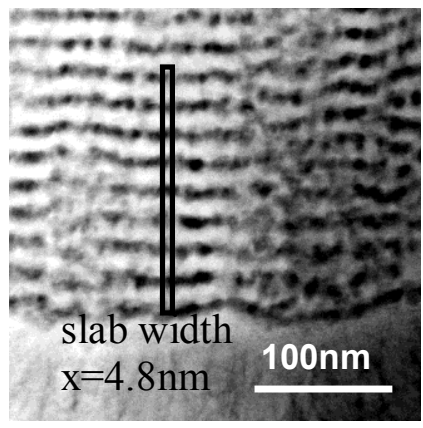

(b)

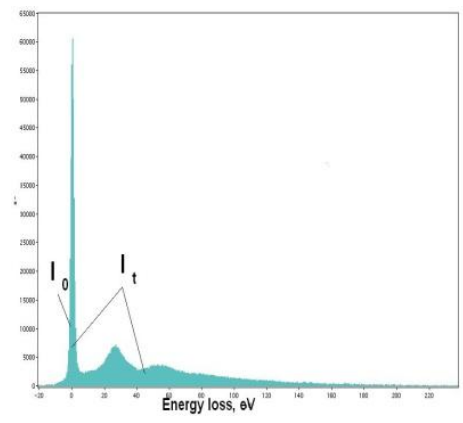

(c)

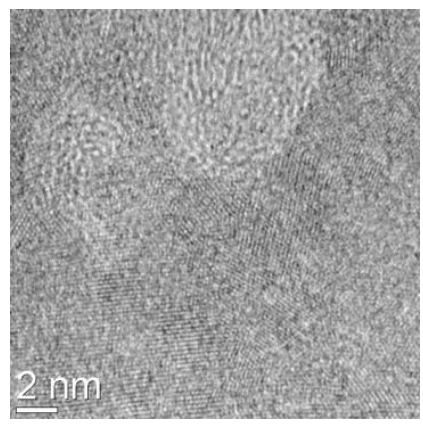

FIG.1 microstructure of the coating using a 2010 FEGTEM. (a) STEM/ADF. (b) EELs of the area of Fig1a. (c) HRTEM.

(a)

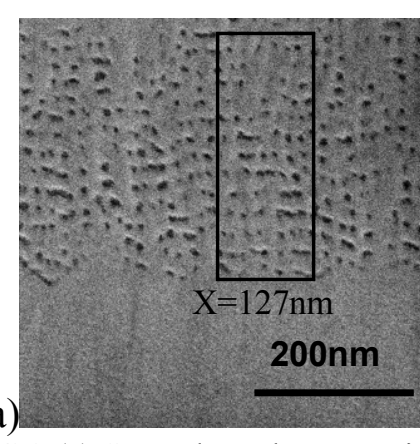

(b)

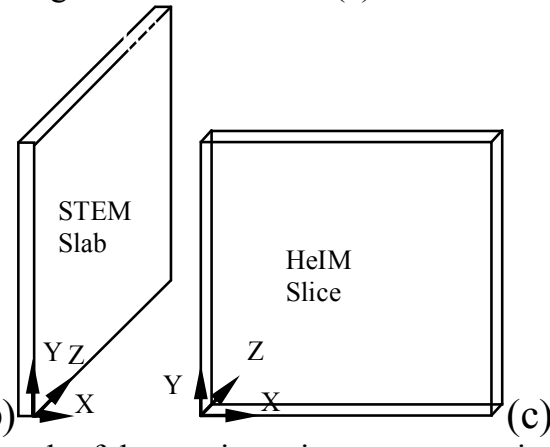

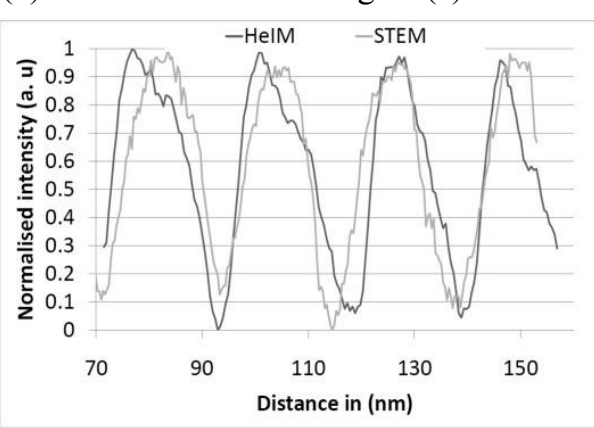

FIG.2 (a) Secondary electron micrograph of the coating microstructure using HeIM. (b) Schematic of the slab in STEM and slice in HeIM to generate intensity profile. (c) Intensity profiles of the selected areas in Fig.1a and Fig.2a. 